\title{
Synthesis of Fibrous Nanoparticle Aggregates by Femtosecond Laser Ablation in Air
}

\section{Bo Tan}

Ryerson University

\section{Krishnan Venkatakrishnan}

Ryerson University

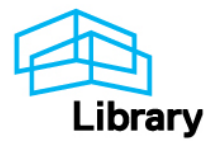




\title{
Synthesis of fibrous nanoparticle aggregates by femtosecond laser ablation in air
}

\author{
Bo $\operatorname{Tan}^{1}$ and Krishnan Venkatakrishnan ${ }^{2 *}$ \\ ${ }^{I}$ Department of Aerospace Engineering, Ryerson University, 350 Victoria Street, Toronto, ON, Canada \\ ${ }^{2}$ Department of Mechanical and Industrial Engineering, Ryerson University, 350 Victoria Street, Toronto, ON, \\ Canada \\ "Corresponding author: venkat@ryerson.ca
}

\begin{abstract}
In this article we report the synthesis of nanoparticles using femtosecond laser ablation with $\mathrm{MHz}$ pulse frequency at room temperature in air. Nanoparticles agglomerate by fusion, and form interweaving fibrous structures that show certain degree of self-assembly. It is found that there is a threshold-like pulse frequency at which fibrous nanoparticle aggregates start to form. The growth mechanism can be explained by existing theories regarding nanoparticle formation through femtosecond laser ablation. The threshold pulse frequency is in good agreement with the time to start nanoparticle formation, which has been derived numerically by previous analyses.
\end{abstract}

(C)2008 Optical Society of America

OCIS codes: (140.3390) Lasers and Laser Optics, (160.4236) Nanomaterials, (220.4241)

Nanostructure fabrication

\section{References and links}

1. M. Ulmann, S. K. Friedlander and A. S.chmidit-Ott, "Nanoparticle formation by laser ablation,” J. Nanopart. Res. 4, 499-509 (2002).

2. S. Li, S. J. Silvers, and M. S. El-Shall, "Surface Oxidation and Luminescence Properties of Weblike Agglomeration of Silicon Nanocrystals produced by a Laser Vaporization-Controlled Condensation Technique," J. Phys. Chem. B. 101, 1794-1802 (1997).

3. B. R. Tull, J. E. Cary, M. A. Sheehy, C. Friend, and E. Mazur, "Formation of silicon nanoparticles and weblike aggregates by femtosecond laser ablation in a background gas," Appl. Phys. A. 83, 341-346 (2006).

4. K. Nishikawa, K. Takano, H. Miyahara, T. Kawamura, A. Okino and E. Hotta, "Nanofractal structure consisting of nanoparticles produced by ultrashort laser pulses," Appl. Phys. Lett. 89, 243112 (2006).

5. T. E. Glover, G. D. Ackerman, R. W. Lee, and D. A. Young, "Probing particle synthesis during femtosecond laser ablation: initial phase transition kinetics," Appl. Phys. B. 78, 995-1000 (2004).

6. F. R. Sushmita and R. K. Thareja, "Simulation of cluster formation in laser-ablated silicon plumes," J. Appl. Phys. 97, 123303 (2005).

7. S. I. Anisimov and B. S. Luk' yanchuk, "Selected problems of laser ablation," Physics-Uspekhi 45, $293-324$ (2002).

8. T. Takiya, I. Umezu, M. Yaga, and M. Han, "Nanoparticle formation in the expansion process of a laser ablated plume,” J. Phys.: Conf. Ser. 59, 445-448 (2007).

9. J Perrière, C Boulmer-Leborgne, R. Benzerga, and S Tricot, "Nanoparticle formation by femosecond laser ablation,” J. Phys. D: Appl. Phys. 40, 7069-7076 (2007).

\section{Introduction}

Nanoparticles have numerous potentials in the fabrication of nanomaterials that applied to microelectronics, biomedical, and photonics devices. For example, they can be used as fillers of polymer composites enhancing the mechanical property [1]. Formation of nanoparticles through laser ablation is an attractive technique owing to the simplicity in equipment configuration and low operation cost. In recent years, intensive investigations have been devoted to nanoparticle formation through femtosecond laser ablation. The rapid superheating caused by the extreme short pulse duration has the potential of opening up new possibilities in the nanoparticle synthesis.

\#103094 - \$15.00 USD Received 21 Oct 2008; revised 22 Nov 2008; accepted 12 Dec 2008; published 15 Jan 2009 
In this article, we report the synthesis of nanoparticles using femtosecond laser ablation of targets of various materials at room temperature in air. Bulk quantity of nanoparticles agglomerate by fusion, and form interweaving fibrous structures that show certain degree of assembly. To the best of our knowledge, this kind of nanostructures has not been reported with femtosecond laser ablation. More interestingly, we found that pulse frequency plays a critical role in the formation of nanoparticle aggregates.

\section{Experiments and results}

In our growth apparatus, a direct-diode-pumped Yb-doped fiber amplified femtosecond laser system was used to ablate the targets. The laser delivers a maximum output power of $15 \mathrm{~W}$ at pulse frequency ranging from $200 \mathrm{kHz}$ to $26 \mathrm{MHz}$ with pulse duration of $200 \mathrm{fs}$. The first tested target was a blank polished Boron doped silicon wafer with $\langle 100\rangle$ crystal orientation. It was held stationary in air at room temperature.

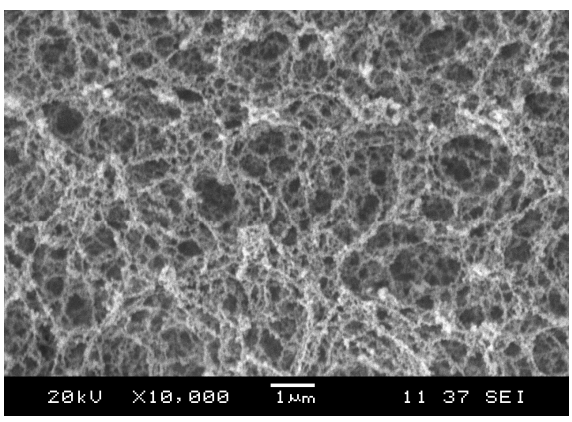

(a) Silicon nanoparticle aggregates found on the sidewall of dice, singulated by laser beam of $515 \mathrm{~nm}$ wavelength, $11 \mathrm{~W}$ laser power at $13 \mathrm{MHz}$ pulse frequency.

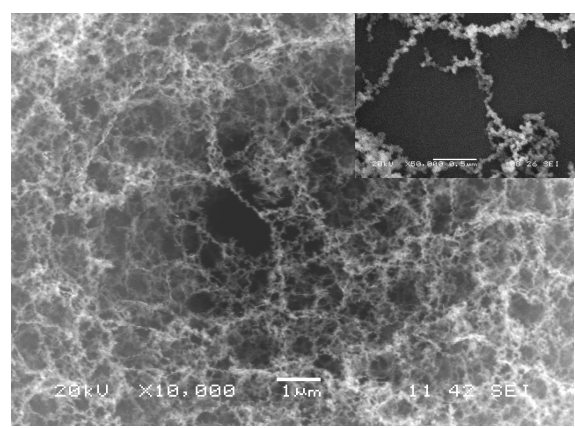

(b) Silicon nanoparticle aggregates formed by laser beam of $1030 \mathrm{~nm}$ wavelength, $11 \mathrm{~W}$ laser power at 13 $\mathrm{MHz}$ pulse frequency and $100 \mu \mathrm{s}$ machining time. The insert shows bridge structure.

Fig. 1. Interweaving fibrous nanoparticle aggregate found after laser ablation of bulk silicon.

We first observed the interweaving fibrous nanoparticle aggregations as a byproduct of silicon wafer singulation at Mega Hertz pulse frequency. After cutting along straight lines on the silicon substrate with multiple cycles, we noticed organized aggregated nanoparticles along the inner sidewalls of the machined grooves (Fig. 1(a)). These fibrous structures look exactly the same as those produced by a Laser Vaporization-Controlled Condensation technique (LVCC) [2]. The conventional LVCC method uses a chamber at high pressure with two plates having large difference in temperature to create a steady convection current, which is enhanced by a heavy carrier gas such as Ar. The discovery of these fibrous nanoparticle aggregates made us hypothesize that at Mega Hertz pulse frequencies, femtosecond laser ablation may result in gas phase condensation.

To confirm this hypothesis, we conducted additional experiments. Arrays of microvias were drilled into the silicon substrate by firing a train of laser pulses. Fibrous nanostructure was formed inside and surrounding the drilled hole. The SEM images show that the fibrous nanostructures were formed with particles of relatively uniformed diameters in the order of 50 $\mathrm{nm}$ (Fig. 1(b)). Electron-induced X-ray fluorescence (EDX) analysis shows that these fibrous structures contain only $\mathrm{Si}$ with a trace of $\mathrm{O}$. The nanostructures have closed-rings and bridges (insert Fig. 1(b)). They are more organized than nanoscale structures obtained with femtosecond laser ablation at repetition rates in the order of 1-10 kHz [3, 4] (Nanoparticles obtained in this regime aggregate by random stacking). Figure 2 shows a chain-like aggregate, with particles fused linely rather than randomly stacked by loose bonds. The circle in Fig. 2 highlights the fusion of two individual particles. The similarity between the particle aggregation generated by our method and the LVCC indicates that the mechanism of the particle-aggregate formation could be related to nucleation and growth during laser ablation. 


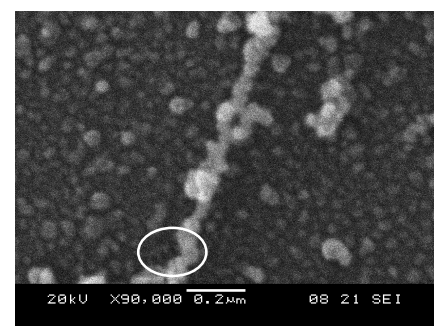

Fig. 2. Fused nanoparticles

The most interesting phenomenon we observed is that the growth of the fibrous nanostructure is pulse-frequency dependent. As shown in Fig. 3(a), at $2 \mathrm{MHz}$, large molten droplets dominate the generated matter, with a small amount of nanoparticle aggregates. As the frequency increases to $4 \mathrm{MHz}$, the amount of molten droplet reduces and that of fibrous nanostructure increases significantly. Similar phenomena were also observed on targets made of graphite, glass, aluminum, tin, lead and composition (tin and lead).

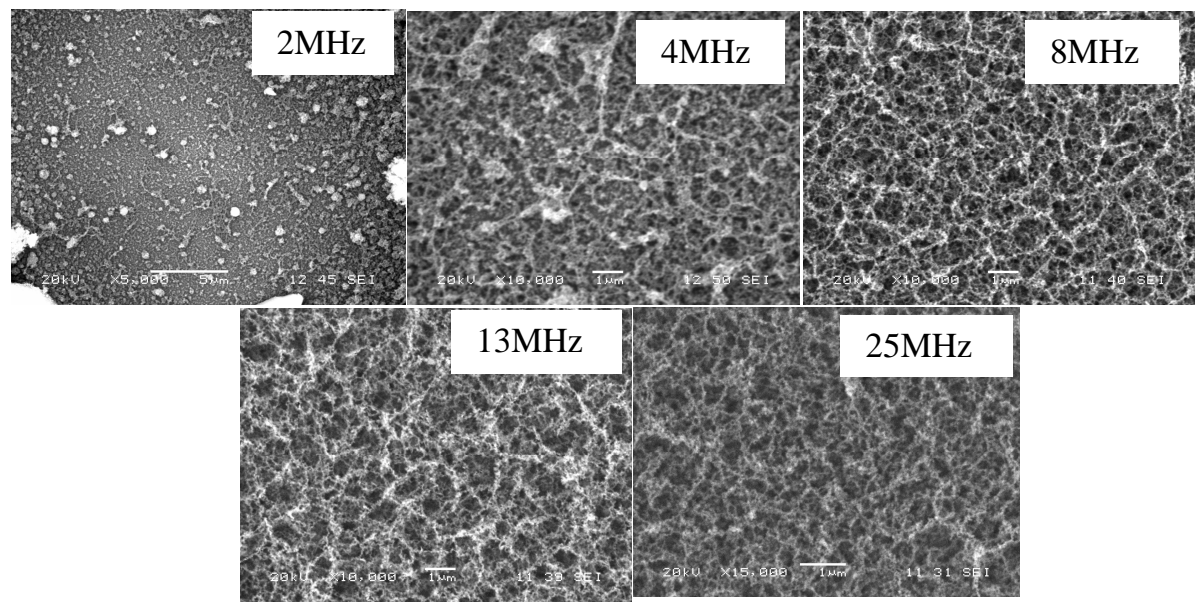

Fig. 3. Fibrous silicon nanostructures formed by laser beam of $1030 \mathrm{~nm}$ wavelength, $200 \mathrm{fs}$ pulse width and $11 \mathrm{~W}$ laser power at various pulse frequencies.

Figure 4 presents fibrous nanostructures generated from targets made of graphite, aluminum, lead and tin. Figure 4(e) shows fibrous nanostructures generated from composite of tin, indium, lead, and bismuth. BK7 glass was also tested for the fibrous nanostructure growth. The area under the target was converted to fibrous nanostructures and is confined within a clearly defined boundary as shown in Fig. 4(f). EDX test shows that the composition of the nanostructures formed from glass respects the BK7 glass composition. It is reasonable to deduce that fibrous nanoparticle aggregations could be generated from GaAs and InP under similar conditions, since these elements are in Group XIII and Group XIV and have similar chemical properties as those already tested in our experiments. Other materials, such as, gold, titanium, copper and nickel were also ablated. No fibrous nanostructures were found even though ablations did occur because microvias as deep as $50 \mu \mathrm{m}$ formed as the results of irradiation. Experimental results show that fibrous nanostructures forms on graphite, silicon, aluminum, tin and lead, at fluence well below the ablation threshold (SEM images is given in Fig. 5). Apparently, in atmosphere environment the fibrous nanostructures formation occurs only for Group XIII and Group XIV materials and their oxides. With current experimental results, we are unable to conclude if the formation is truly material-depend because materials 
like gold and titanium may form fibrous nanostructures under a different ambient environment.

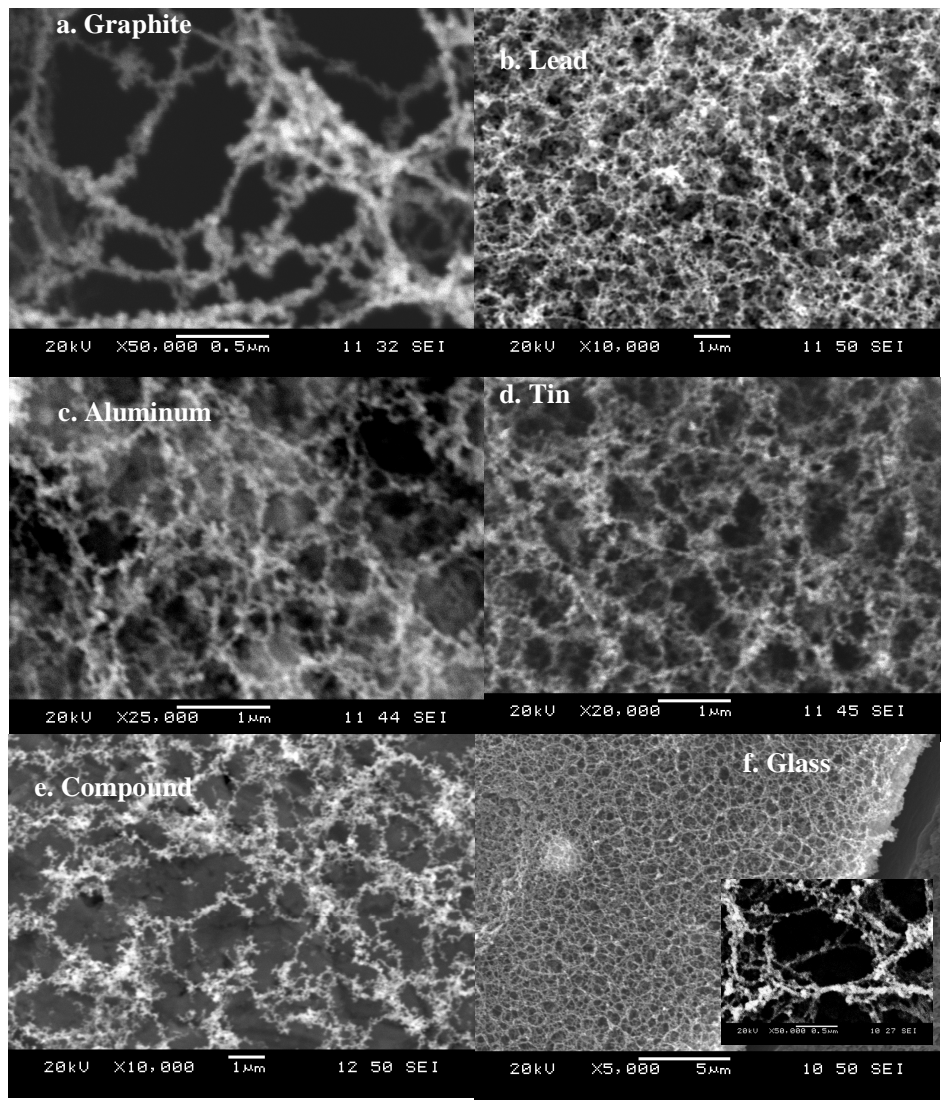

Fig. 4. Fibrous nanostructures formed from materials from Group XIII and Group XIV materials. Laser beam wavelength is $515 \mathrm{~nm}$, pulse frequency is $13 \mathrm{MHz}$, and laser power is $11 \mathrm{w}$. Compound target material consists of tin, indium, lead, and bismuth.

Previous study of femtosecond laser ablation of matter by T. E. Glover [5] shows that critical local expansion dynamics dominates the initial phase transition, leading to the direct fragmentation and ejection of the molten target material. The liquid-phased fragments, of size less than $1 \mathrm{~nm}$, reach to solidification temperature within $50 \mathrm{ps}$. This solidification time is nearly three orders of magnitude shorter than that of a thermal vapor condensation process. The rapid solidification of the ejected liquid fragments suggests a highly undercooled material phase. The subsequent growth of the solid fragments will form nanoparticles of size in the order of $10 \mathrm{~nm}$. Agglomerates will also grow as a result of collisions between individual nanoparticles through chemical bonds and attraction forces at the interfaces. The nanoparticle growth will come to a haul when the density reduces to the equilibrium concentration or the particles temperature reduces to equilibrium.

The formation of nanoparticles is more complex in the presence of background gas; Plume expansion and collisions with gas atoms have to be considered. Prior studies have demonstrated that the initial expansion of the plume is independent of the background pressure and proceeds as vacuum expansion. The assumption of vacuum expansion is no more valid when the pressure in the plume approaches to gas pressure. From this moment, the collision between species in the ablated plume and the gas atoms plays a role in the formation 
of nanoparticles and aggregates. It is also noteworthy that not all species in the plume collide with the atmosphere atoms, only those in the thin interface layer do. Since most atoms in air(71\% Nitrogen and $21 \%$ oxygen) have mass comparable to that of silicon atoms, the collisions lead to both inward and outward diffusion[6].

The density of the ablated species decreases as the pulse expands. Growth of particles and agglomerates stops when the density reduces to the equilibrium concentration. In the case of multiple-shot ablation, as the case of our experiments, if the successive pulses arrive before the density drops below the equilibrium concentration, a constant flux of newly formed particles can be maintained, resulting in a steady growth of the nanoparticles and agglomerates.

The kinetic energy of the rapid expansion of vapor produced by laser ablation has been analyzed numerically [7-8]. It has been found that particles start to form less than $1 \mu$ s after the laser irradiation. For example, Anisimov [3] calculated that germanium vapor starts to condense $0.75 \mu \mathrm{s}$ after irradiation in a vacuum. With buffer gas, the plume expansion will be slowed down. Therefore, the time to particle formation will be delayed. Simulation result by Takiya[8] shows that the density of nanoparticles start to build up after $0.901 \mu$ s in a silicon plume expanding in a buffer gas. The $0.901 \mu$ s corresponds to a pulse frequency around 1.1 MHz. This is at the same magnitude with the pulse frequency of $2 \mathrm{MHz}$ at which fibrous nanoparticle aggregates start to appear on the silicon target. A recent experiment conducted $\mathrm{MgO}$ sample [9] showed that nanoparticle density reaches to peak about $2 \mu \mathrm{s}$ after femtosecond laser irradiation. This time scale also suggests a pulse frequency in the order of Mega Hertz.

The threshold-like pulse frequency was observed for all the tested materials that produce fibrous nanostructures. For instance, fibrous agglomerate starts to show up at $1 \mathrm{MHz}$ for graphite. The fact that graphite forms aggregates at lower pulse frequency than silicon does is in a good agreement with the theoretical analysis of the onset time of nanopartilce formation in ambient gas[6]. Since carbon atoms are lighter than silicon atoms, they need more time to cluster and carbon plume expands slower than silicon plume. Therefore, constant flux of newly formed nanoparticles can be sustained at a lower pulse frequency.

As aforementioned, micron-sized molten droplets coexist with nanoscale fibrous structures at pulse frequencies close to the threshold pulse frequency. The amount of large molten droplets reduces with the increase of pulse frequency and disappears after $13 \mathrm{MHz}$. This can be explained by the three laser fluence thresholds associated with the emission of ablation species: atomic species, clusters, and droplets[9]. The threshold to form droplets is much higher than those to form atomic species and clusters. As laser power was kept constant in our experiments, pulse energy reduces with the increase of pulse frequency, from $5.5 \mu \mathrm{J}$ at $2 \mathrm{MHz}$ to $0.4 \mu \mathrm{J}$ at $26 \mathrm{MHz}$. With a constant laser spot size, fluence reduces at the same rate. At low pulse frequency the fluence is high, resulting in emission of all three species; while at higher frequency, the fluence is above the threshold of the emission of atomic species and clusters but lower than the threshold of droplet emission. Therefore, nanostructures generated at higher pulse frequencies are free of molten droplets.

Further study on the effect of laser fluence on aggregation growth shows that the size of aggregates increases with the increase in laser fluence. Figure 5 presents SEM images of aggregates produced at increasing laser fluence. At low fluence that does not induce material break-down, we observed tree-branch-like aggregates of lengths in micron range (Fig. 5(a)). The aggregate tends to grow more in one direction, forming wire-like structures with branches. As the laser fluence increases, the length of the wire increases and more branches sprout along the wire. Finally, a web-like interweaving nanostructure is formed.

We noticed that the fibrous nanostructures have relatively uniformed diameters and did not observe a wide range of variation in size distribution. This agrees with the characteristics of nanoparticle formation through homogenous nucleation, which tends to generate monosized nanoparticles.

The production of short, chain-like nanoparticle aggregates suggests that localized growth of nanochain-like structure can be obtained at very low laser fluence. Figure 5(d) shows

\#103094 - \$15.00 USD Received 21 Oct 2008; revised 22 Nov 2008; accepted 12 Dec 2008; published 15 Jan 2009 
chain-like nanostructures created along a line at fluence of $0.3 \mathrm{~W}$, as the laser beam rastered across the surface of a silicon substrate. This enables growth of fibrous nanostructure at selected locations by controlling the laser fluence and scanning direction.

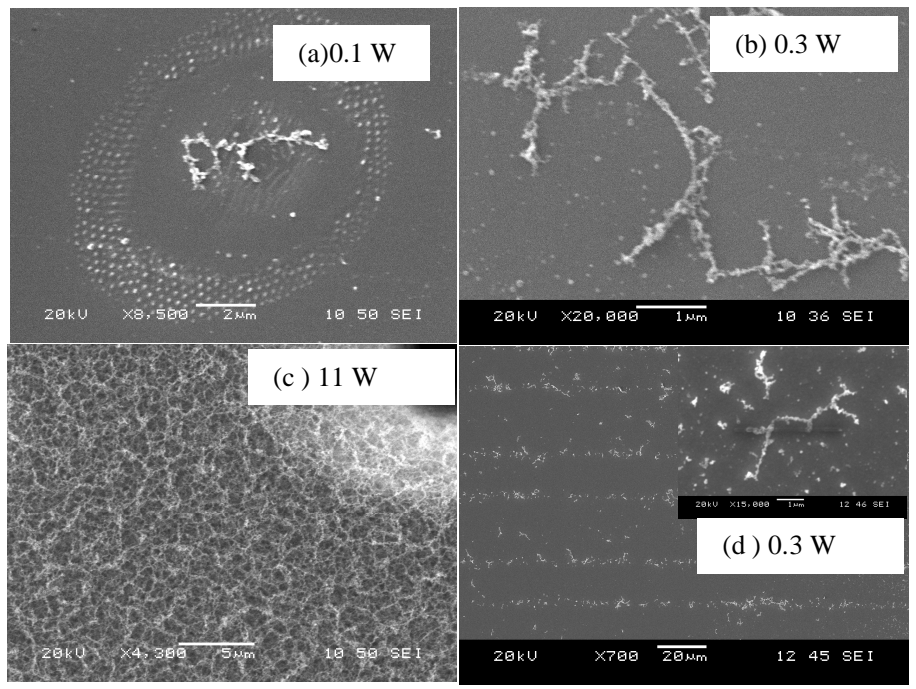

Fig. 5. Effect of laser fluence. Laser parameters: $1030 \mathrm{~nm}$ wavelength at $13 \mathrm{MHz}$ pulse frequency

\section{Summary}

In this research, we observed a new phenomenon associated with nanoparticle formation through femtosecond laser ablation. At Mega Hertz pulse frequency, interweaving fibrous nanostructures form by the agglomeration of nanoparticles and their aggregates. The fibrous nanostructure formation is found to occur only for Group XIII and Group XIV materials under current experimental conditions. A distinct characteristic of the fibrous nanostructures is that particles are fused and the agglomeration shows certain degree of organization, unlike the random stacking of particles observed at femtosecond laser ablation at pulse frequency in $\mathrm{kHz}$ and $\mathrm{Hz}$ regime. At laser fluence lower than ablation threshold, chainlike nanoparticle aggregates can be produced at designated locations. It is also found that the pulse frequency at which the particle aggregations form is in agreement with the theoretically calculated time to start nanoparticle formation. Existing theories of nanoparticle formation by femtosecond ablation were used to explain some of the observed phenomena. More investigations need to be done in order to fully understand the mechanisms of this new phenomenon.

The present discovery may open up new possibilities in nanomaterial syntheses. For example: it could be used to fabricate indium-tin-oxide nanofibers, a material currently being investigated for applications in high-efficiency solar cells; ablation of bioactive glass may lead to nanoporous scaffolds for bone tissue engineering; the fibrous nanostructues could be used as fillers for polymer nanocomposites. Since the formation of the fibrous nanostructure occurs in air at room temperature, this method has the potential of leading to processes for mass production.

\section{Acknowledgment}

This research is funded by Natural Science and Engineering Research Council of Canada. The authors appreciate Mr. Qiang Li for his tremendous efforts in sample characterization. 